\title{
Controlling Nonequilibrium Phonon Populations in Single-Walled Carbon Nanotubes
}

\author{
Mathias Steiner, Huihong Qian, ${ }^{\dagger}$ Achim Hartschuh, ${ }^{\dagger}$ and Alfred Johann Meixner* \\ Institut für Physikalische und Theoretische Chemie, Universität Tübingen, \\ 72076 Tübingen, Germany
}

Received March 24, 2007; Revised Manuscript Received May 31, 2007

\section{ABSTRACT}

We studied spatially isolated single-walled carbon nanotubes (SWNTs) immobilized in a quasi-planar optical $\lambda / 2$-microresonator using confocal microscopy and spectroscopy. The modified photonic mode density within the resonator is used to selectively enhance or inhibit different Raman transitions of SWNTs. Experimental spectra are presented that exhibit single Raman bands only. Calculations of the relative change in the Raman scattering cross sections underline the potential of our microresonator for the optical control of nonequilibrium phonon populations in SWNT.

The control of the vibrational degrees of freedom or, in other words, the phonon populations in molecular nanostructures like single-walled carbon nanotubes (SWNT) is one of the major goals in nanoscience. ${ }^{1,2}$ The electronic structure of SWNTs, see, e.g., refs 3 and 4, is highly susceptible to electron-phonon interactions, and nonequilibrium phonon populations can drastically alter device properties. Hot phonon electron scattering, for example, is known to saturate the charge transport along SWNTs. ${ }^{5-14}$ As a result, the application of SWNTs in electronic and optoelectronic devices, e.g., ${ }^{15}$ could be limited. In the following, we demonstrate the potential of a planar microresonator for the optical control of nonequilibrium phonon populations in SWNTs, i.e., phonon populations that are driven out of thermal equilibrium as imposed by the modified photonic mode density in the presence of the microresonator. Moreover, we describe a strategy for controlling hot phonons in electrically driven SWNTs.

The efficiency of spontaneous radiative transitions in optical $\lambda / 2$ microresonators, i.e., resonators with mirror spacings $L$ of one-half emission wavelength $\lambda$ of embedded emitters, is determined by the local photonic mode density $\rho_{\text {cav }}(L)$ that can be enhanced and inhibited with respect to the corresponding value $\rho_{0}$ in free (nonconfined) space; see, e.g., ref 16 and references therein. Accordingly, both luminescence and Raman scattering processes of embedded emitters can be controlled by enhancement and inhibition of the spontaneous emission rate $\Gamma_{\text {cav }}{ }^{17,18}$ or the Raman

\footnotetext{
* Corresponding author. E-mail: alfred.meixner@uni-tuebingen.de.

† Present address: Department für Chemie und Biochemie and CENS, Ludwig-Maximilians-Universität München, 81377 München, Germany.
}

scattering cross section $\sigma_{\text {cav }},{ }^{19,20}$ respectively, since both scale with the photonic mode density following $\Gamma_{\mathrm{cav}}(L) / \Gamma_{0}=\sigma_{\mathrm{cav}^{-}}$ $(L) / \sigma_{0} \propto \rho_{\text {cav }}(L) / \rho_{0} .{ }^{21}$ In Raman scattering processes the radiative transition is accompanied by the creation (destruction) of a phonon. Hence, it should be possible to populate (depopulate) a specific phonon mode in an embedded SWNT using microresonator-controlled Stokes (anti-Stokes) Raman scattering.

In Figure 1a, a schematic of the microresonator is shown. The sample was prepared from a poly(methyl methacrylate) (PMMA)/dichloroethane solution containing purified SWNT with a diameter distribution ranging from 0.8 to $1.8 \mathrm{~nm}$ (BuckyUSA). A droplet of the solution was enclosed between two silver mirrors. After evaporation of the dichloroethane, the PMMA film fixed the two mirrors and exhibited immobilized SWNT structures spatially separated in the order of $1 \mu \mathrm{m}$. Light passing the microresonator in the $\lambda / 2$-regime obeys the transmission condition

$$
L(x, y)=\left(1-\frac{\sum_{i} \Delta \phi_{i}\left(d_{i}, \vartheta, \lambda\right)}{2 \pi}\right) \frac{\lambda(x, y)}{2 n_{\mathrm{pol}} \cos \vartheta}
$$

Here, $\Delta \phi_{i}$ denotes the phase change due to reflection at the respective silver mirror $i=1,2$ with thickness $d_{i}\left(d_{1}=\right.$ $30 \mathrm{~nm}, d_{2}=60 \mathrm{~nm}$; reflectivities $R_{1}=0.7, R_{2}=0.9$ at $\lambda=$ $532 \mathrm{~nm}$ for perpendicular incidence) and $\sum_{i} \Delta \phi_{i}$ was calculated to be around 1.3 in the present case. The refractive index of the intracavity medium is $n_{\text {pol }}(=1.49$ for PMMA). The incidence angle of a parallel light beam with respect to the $z$-axis shown in Figure 1 is given by $\vartheta$. If the transmission condition eq 1 is fulfiled for $\vartheta=0$, the corresponding 


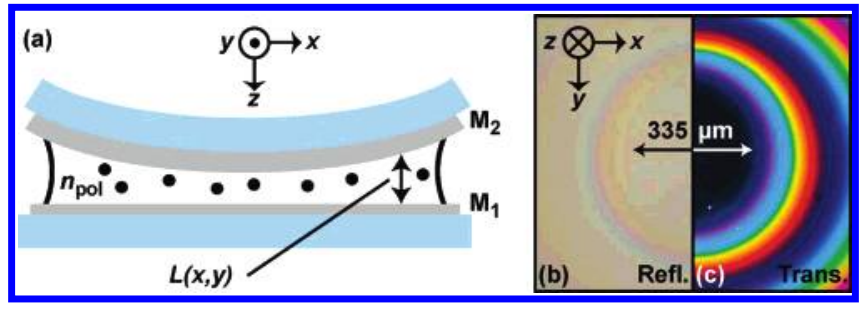

Figure 1. Optical microresonator doped with single-walled carbon nanotubes (SWNTs). (a) The sample consists of two mirrors, $\mathbf{M}_{1,2}$, prepared by evaporating silver films onto glass coverslips. The mirrors are separated by a layer of a transparent polymer (PMMA) with varying thickness enclosing homogenously distributed and randomly oriented SWNTs that are indicated by dots. Optical micrographs show the microresonator center illuminated with white light in (b) reflection and (c) transmission on $\mathrm{M}_{1}$. The local mirror spacing $L(x, y)$ is extracted from (c) using eq 1 and served to calculate the normalized Raman scattering cross sections $\sigma_{\text {cav }}(L) /$ $\sigma_{0}$ of embedded SWNTs shown in Figure 4.

photonic mode is called an on-axis resonance; in the case $\vartheta$ $>0$, it is called off-axis resonance. Hence, eq 1 yields the local mirror spacing $L(x, y)$ from the measured transmitted wavelength $\lambda(x, y)$ for $\vartheta=0$. As can be seen from the concentric interference pattern shown in parts $b$ and $c$ of Figure 1, the mirrors are slightly inclined with respect to each other and allow addressing SWNTs for different $L(x, y)$ and, hence, local photonic mode densities $\rho_{\text {cav }}(L)$. The mirror spacing variation $\Delta L(x, y) / \Delta x, y \cong 10^{-3}$ ensures a planar resonator geometry within the focal diameter $(<0.5 \mu \mathrm{m})$ of the microscope objective. A detailed description of the microresonator and its applications on single molecule fluorescence microscopy have been reported before. ${ }^{22}$ For confocal microscopy and spectroscopy, a HeNe laser light beam $\left(\lambda_{\text {exc }}=632.8 \mathrm{~nm}\right)$ providing excitation powers in the range of $0.5 \mathrm{~mW}$ was tightly focused on either the reference sample or the microresonator using an immersion oil microscope objective $(100 \times, \mathrm{NA}=1.25)$. The samples were raster scanned with respect to the fixed microscope objective, and the Raman-scattered light was separated from the laser excitation light using a suitable holographic notch filter and guided into a grating spectrograph. The exposure time for the Raman spectra shown in Figure $2 b$ and Figure $3 b$ was 5 and $0.195 \mathrm{~s}$, respectively.

As reference, a confocal optical Raman microscopy image of spatially isolated SWNT structures homogeneously distributed in a thin film of PMMA is shown in Figure 2a. The Raman spectrum (see Figure 2b) measured on an isolated bright spot reveals the phononic structure in SWNT that is dominated by contributions from the radial breathing mode vibration (RBM), the disorder-induced vibration (D), its overtone $\left(\mathrm{G}^{\prime}\right)$, and the $\mathrm{CC}$ stretch vibration $(\mathrm{G})^{4,23}$ Without microresonator, the relative intensities of the different Raman bands are determined by intrinsic properties of the nanotube such as the phonon-mode-specific electron-phonon coupling and the joint density of states. In addition, resonance Raman scattering that occurs if the incident or scattered photon energy is in resonance with an electronic transition of the nanotube can enhance the signal essentially and is the reason why single nanotubes can be observed in the first place. ${ }^{23}$ The resonance Raman condition is an intrinsic property of

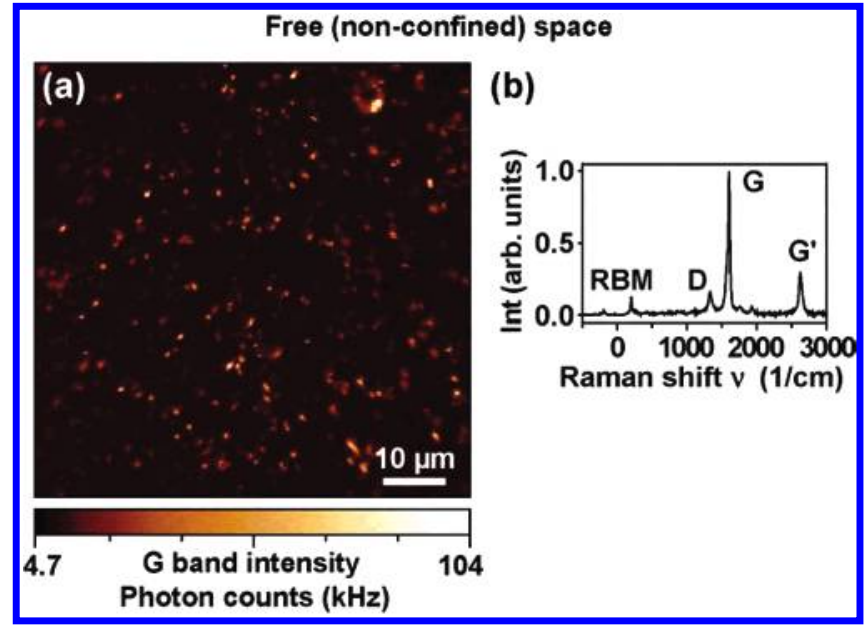

Figure 2. Raman microscopy and spectroscopy of SWNTs dispersed in a thin film of PMMA: (a) confocal Raman microscopy image; (b) Raman spectrum taken from one of the bright spots in (a).

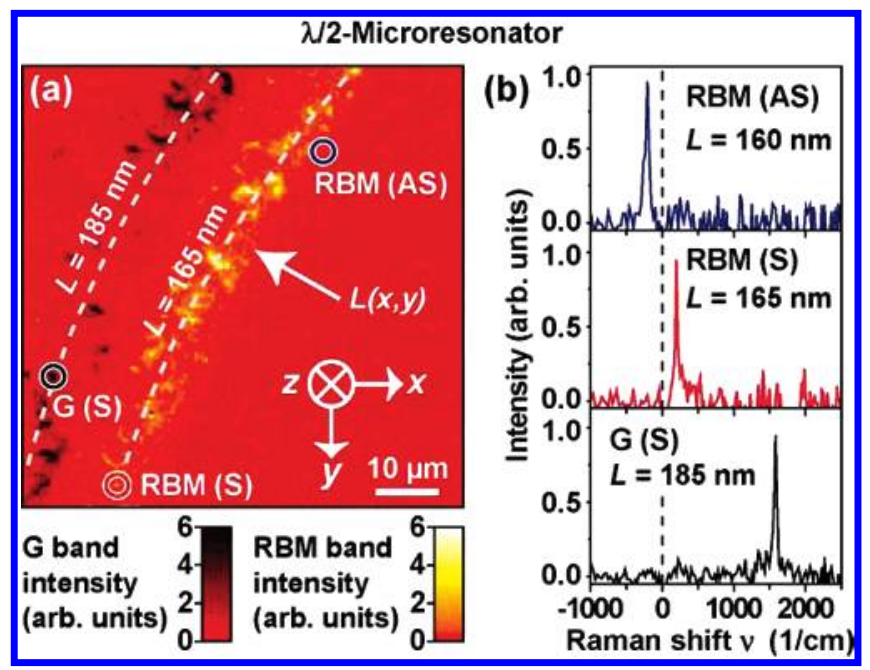

Figure 3. Raman microscopy and spectroscopy of SWNT embedded in an optical $\lambda / 2$ microresonator. (a) Confocal Raman microscopy image of the same sample as shown in Figure 2, but enclosed between the mirrors of a $\lambda / 2$ microresonator. Strong scattering intensities of embedded SWNT are obtained where the G band (dark spots) and the RBM band (bright spots) are on resonance with the local photonic mode density $\rho_{\text {cav }}(\lambda, L)$. The direction of increasing mirror spacing $L(x, y)$ is indicated by the arrow and the curvature of the rings of equal $L$ is indicated by the dashed lines. (b) Representative microresonator-controlled Raman spectra of SWNTs measured at the positions marked in (a) by colored rings [blue, RBM band (anti-Stokes); red, RBM band (Stokes); black, G band (Stokes)]. To allow data acquisition times of less than $200 \mathrm{~ms}$ during the image scan, a spectral binning was used and the Raman spectra were acquired with reduced spectral resolution $\left(\Delta v \cong 50 \mathrm{~cm}^{-1}\right)$.

the nanotube and is not related to the resonance between the light emitted by the nanotube and the photonic mode density of the resonator discussed in the following.

Using the same PMMA-SWNT solution as the intracavity medium between the mirrors of the $\lambda / 2$-microresonator, we observed completely different confocal Raman microscopy images. In particular, we found that the scattering intensities of the phonon modes in SWNT depend on the local mirror spacing $L(x, y)$ and hence the photonic mode density $\rho_{\text {cav }}(L)$. 
To illustrate this spatial dependency, the Raman image shown in Figure 3a was constructed in the following way: For each pixel, a Raman spectrum was acquired and the intensities of the RBM band and the $\mathrm{G}$ band were integrated independently and shown in different color scales. As a result, two sectors of spatially separated concentric rings of equal $L$ appeared: The inner ring is formed by bright spots originating from SWNT showing strong RBM intensities, the outer ring consists of dark spots that stem from SWNT showing strong $\mathrm{G}$ intensities.

Representative microresonator-controlled Raman spectra taken for the two rings of constant $L$ are shown in Figure $3 \mathrm{~b}$ together with a spectrum measured for the $L$ corresponding to the RBM anti-Stokes transition. Each spectrum of SWNT consists of a single Raman band corresponding to the Raman transition that is on resonance with $\rho_{\text {cav }}(L)$. The absence of a detectable contribution from the $\mathrm{G}$ band in the measured Raman spectra of all SWNT within the bright inner ring in Figure $3 \mathrm{a}$ is the experimental proof for the inhibition of the optical population of the phonon modes associated with the $\mathrm{G}$ band by at least 1 order of magnitude for the broad $(n, m)$ distribution of the sample material.

The control of the spontaneous emission rate utilizing planar optical microresonators is well-understood, ${ }^{19,21,24}$ and we applied the description successfully to single molecule fluorescence. ${ }^{22}$ We now quantify the enhancement and inhibition for different Raman transitions and discuss how the generation of microresonator-controlled nonequilibrium phonon populations in SWNT is achieved. In Figure 4, the energy level diagram of microresonator-controlled Raman scattering on SWNT is shown together with the three representative spectra from Figure $3 \mathrm{~b}$. Shown below is $L$ determined according to eq 1 and expressed in terms of the Raman shift $v$ with respect to the fixed excitation wavelength $\lambda_{\text {exc }}=633 \mathrm{~nm}$. To compare the modified scattering probabilities, we calculated the normalized microresonatorcontrolled Raman scattering cross sections $\sigma_{\text {cav }}(L) / \sigma_{0}$ for the three transitions and plotted them as a function of the Raman shift $v$. A comparison of the resulting curves for a given $L$ provides a direct measure for the efficiency of the microresonator-controlled optical population (depopulation) of specific phonon modes with respect to nonconfined space. The $\sigma_{\mathrm{cav}^{-}}$ $(L) / \sigma_{0}$ curves shown in Figure 4 represent the coupling of Raman transitions of SWNT to the on-axis resonance $(\vartheta=$ 0 in eq 1$)$ as well as the off-axis resonances $(\vartheta>0)$ provided by our planar microresonator.

Efficient band-selective enhancement and inhibition can only be achieved for $L$ below $160 \mathrm{~nm}$, i.e., below the onaxis resonance of the excitation light (Raman shift $v \leq$ $\left.0 \mathrm{~cm}^{-1}\right)$. Here, the ratio between the normalized scattering cross sections is RBM (anti-Stokes)/RBM (Stokes)/G (Stokes) $=12 / 5 / 1$. This ratio determines the measured microresonatorcontrolled Raman spectrum at $L=160 \mathrm{~nm}$ where we only observe anti-Stokes scattering of the RBM (blue spectrum). Accordingly, the population of the RBM phonon is optically driven out of thermal equilibrium $\left(T_{\text {sample }} \approx 300 \mathrm{~K}\right)$. This result represents the basis for the control of nonequilibrium phonon populations in SWNT.

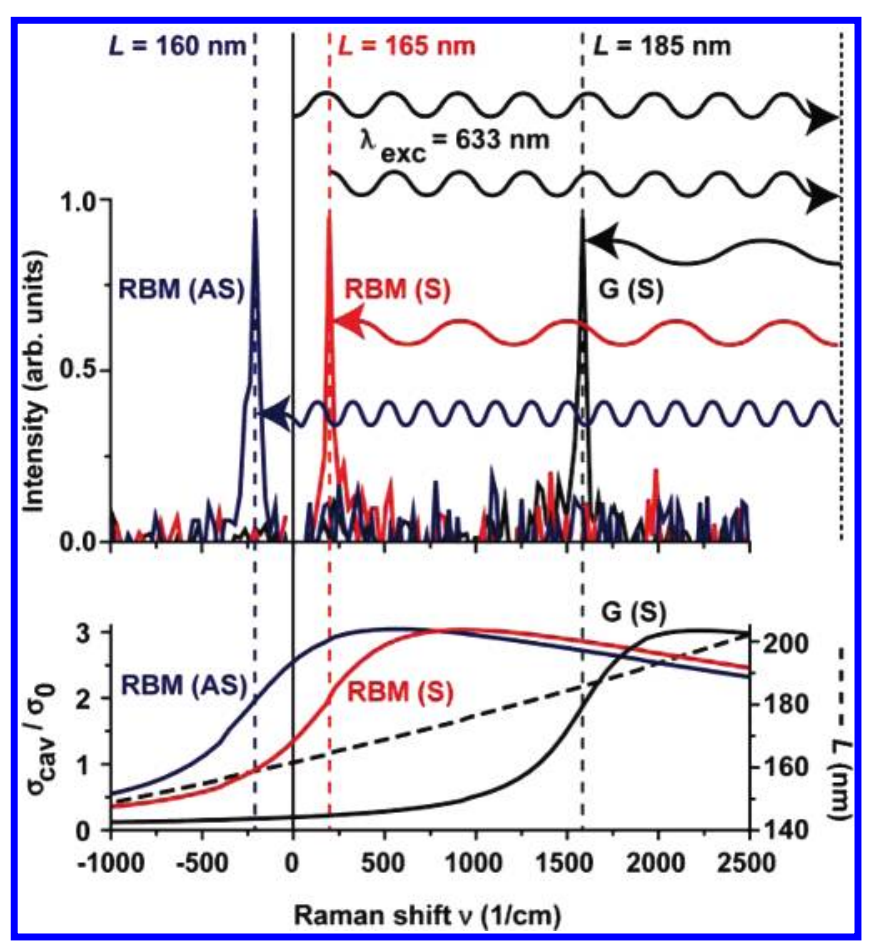

Figure 4. Microresonator-controlled Raman transitions of SWNTs. Energy level diagram, measured spectra and calculated normalized scattering cross sections $\sigma_{\text {cav }}(L) / \sigma_{0}$ are shown for three representative transitions [blue, RBM (anti-Stokes); red, RBM (Stokes); black, G (Stokes)]. The intersections between the dashed $L$ curve and the dashed vertical lines mark the resonant $L$ values for the measured spectra. A comparison of the values taken from the $\sigma_{\text {cav }}(L) / \sigma_{0}$ curves for the three resonant $L$ values provides the enhancement (inhibition) ratios for the nonequilibrium phonon populations associated with the RBM band and the $\mathrm{G}$ band, respectively.

In contrast, we find that for $L$ around $185 \mathrm{~nm}$ the scattering cross sections $\sigma_{\text {cav }}$ of all Raman bands [blue, RBM (antiStokes); red, RBM (Stokes); black, G (Stokes)] are enhanced as compared to the respective cross sections $\sigma_{0}$ without resonator by a factor of 2 to 3 . We obtain the ratio between the normalized cross sections to be RBM (anti-Stokes)/RBM $($ Stokes $) / \mathrm{G}($ Stokes $)=1.4 / 1.3 / 1$. We note that the excellent spectral isolation of the $\mathrm{G}$ band observed in Figure $3 \mathrm{~b}$ for $L$ $=185 \mathrm{~nm}$ results only from the reduced detection efficiency of the RBM bands. At this position, radiation from the RBM transitions is coupled to off-axis resonances that effectively form waveguide modes between the resonator mirrors and thus cannot be detected.

We now discuss the potential of the $\lambda / 2$ microresonator for the control of the phonon population in a SWNT, e.g., removing vibrational energy from a SWNT that is initially in thermal equilibrium with its local environment. Due to the large number of atoms, SWNTs offer various degrees of freedom and efficient internal relaxation paths that compete with the radiative relaxation. These internal relaxation channels can of course not be controlled by the microresonator. In particular, the extremely low photoluminescence quantum yield for embedded SWNTs on the order of $10^{-3}$ further deteriorates the energy balance: Almost every photon absorbed by SWNTs will be converted into heat, i.e., phonon excitations. 
The suppression of specific phonon populations, on the other hand, is conceivable, and our results indicate a strategy for controlling hot phonons in electrically driven SWNTs that are in focus of device applications. Here, the phonon populations associated with the $\mathrm{G}$ band, i.e., the zoneboundary and optical phonon modes in SWNTs between $\hbar \Omega$ $\cong 0.15$ and $0.2 \mathrm{eV}$, are limiting the charge transport along SWNTs by electron phonon scattering. ${ }^{12,13}$ To efficiently suppress the phonon population corresponding to the $\mathrm{G}$ band by means of the $\lambda / 2$ microresonator, two resonance conditions have to be met: First, the laser energy has to match the resonance Raman condition of the anti-Stokes transition, i.e., $E_{i i}-\hbar \omega_{\mathrm{G}}$, of a specific SWNT with transition energy $E_{i i}{ }^{25,26}$ Second, the anti-Stokes transition has to fulfill also the photonic resonance condition of the microresonator determined by $\rho_{\text {cav }}(\lambda, L)$. We expect a dynamical equilibrium between the phonon bath and the electrically induced nonequilibrium phonon population that is reduced by the enhanced anti-Stokes Raman transition depending on the laser excitation intensity. From our calculations we obtain the maximum ratio between the respective normalized scattering cross sections to be $\mathrm{G}$ (anti-Stokes)/G (Stokes) = 20. This mode-selective effect could be probed by measuring the current-voltage characteristics for charge transport along an individual SWNT under high-field conditions. ${ }^{6-14}$ As a result, we expect the current saturation level to be elevated with respect to the corresponding value in absence of the microresonator due to a net depopulation of hot optical phonons that are predicted to reach temperatures of up to several thousand kelvin. ${ }^{12,13}$ Additionally, current limitations induced by defect-related ( $\mathrm{D}$ band) phonons could be efficiently removed by a second laser that is used to depopulate the Raman-active second-order $\mathrm{G}^{\prime}$ band phonons. ${ }^{3,4}$ We believe that the optical control of the hot phonon population in SWNT with a modified photonic mode density induced by integrated micropost and whispering gallery mode resonators, see, e.g., ref 16 and references therein, is a promising way to surpass physical limitations in SWNT devices.

For larger mirror spacing around $L=280 \mathrm{~nm}$, we observed microresonator-controlled photoluminescence from semiconducting SWNTs. ${ }^{27}$ Since the use of SWNTs as photonic elements is still limited by the poor PL quantum yield, utilizing $\lambda / 2$ microresonators can help to improve their PL efficiency by enhancing the radiative exciton decay rate. ${ }^{28}$ Because of the rapid development of optoelectronic devices based on SWNTs, ${ }^{29,30}$ electrically driven SWNT lasers based on microresonator-enhanced luminescence or Raman scattering seems to come into reach.

Acknowledgment. We thank A. Jorio (UFMG, Belo Horizonte, Brazil) and A. K. Swan (BU, Boston, USA) for fruitful discussions and D. Schupp for help with sample preparation. Financial support was given by the Deutsche Forschungsgemeinschaft (Me 1600/6-1/2).
Supporting Information Available: An outline of the theoretical modeling of the microresonator-controlled Raman scattering cross section $\sigma_{\text {cav }}(L) / \sigma_{0}$. This material is available free of charge via the Internet at http://pubs.acs.org.

\section{References}

(1) LeRoy, B. J.; Lemay, S. G.; Kong, J.; Dekker, C. Nature 2004, 432 371-374.

(2) Mann, D.; Pop, E.; Cao, J.; Wang, Q.; Goodson, K.; Dai, H. J.Phvs. Chem. B 2007, 110, 1502-1505.

(3) Dresselhaus, M. S.; Dresselhaus, G.; Avouris, P. Carbon Nanotubes: Synthesis, Structure, Poperties and Applications; Topics on Applied Physics Springer: Heidelberg, 2001; Vol. 80.

(4) Reich, S.; Thomsen, C.; Maultzsch, J. Carbon Nanotubes; WileyVCH: Weinheim, 2004.

(5) Dai, H.; Wong, E. W.; Lieber, C. M. Science 1996, 272, 523-526.

(6) Yao, Z.; Kane, C. L.; Dekker, C. Phys. Rev. Lett. 2000, 84, 29412944

(7) Javey, A.; Guo, J.; Wang, Q.; Lundstrom, M.; Dai, H. Nature 2003, $424,654-657$

(8) Javey, A.; Guo, J.; Paulsson, M.; Wang, Q.; Mann, D.; Lundstrom, M.; Dai, H. Phvs. Rev. Lett. 2004, 92, 106804.

(9) Park, J.-Y.; Rosenblatt, S.; Yaish, Y.; Sazonova, V.; Üstünel, H.; Braig, S.; Arias, T. A.; Brouwer, P. W.; McEuen, P. L. Nano Lett. 2004, 4, 517-520.

(10) Perebeinos, V.; Tersoff, J.; Avouris, P. Phvs. Rev. Lett. 2005, 94, 086802.

(11) Pop, E.; Mann, D.; Cao, J.; Wang, Q.; Goodson, K.; Dai, H. Phys. Rev. Lett. 2005, 95, 155505

(12) Lazzeri, M.; Piscanec, S.; Mauri, F.; Ferrari, A. C.; Robertson, J. Phys. Rev. Lett. 2005, 95, 236802.

(13) Lazzeri, M.; Mauri, F. Phvs. Rev. B 2006, 73, 165419.

(14) Perebeinos, V.; Avouris, P. Phvs. Rev. B 2006, 74, 121410(R).

(15) Avouris, P.; Chen, J. Mater. Todav 2006, 9, 46-54.

(16) Vahala, K. J. Nature 2003, 424, 839-846.

(17) Purcell, E. M. Phys. Rev. 1946, 69, 681.

(18) Kleppner, D. Phvs. Rev. Lett. 1981, 47, 233-236.

(19) Cairo, F.; De Martini, F.; Murra, D. Phys. Rev. Lett. 1993, 70, $1413-$ 1416.

(20) Fainstein, A.; Jusserand, B.; Thierry-Mieg, V. Phvs. Rev. Lett. 1995, $75,3764-3767$.

(21) Yokoyama, H.; Ujihara, K. Spontaneous Emission and Laser Oscillation in Microcavities; CRC Press: Boca Raton, FL, 1995.

(22) Steiner, M.; Schleifenbaum, F.; Stupperich, C.; Failla, A. V.; Hartschuh, A.; Meixner, A. J. ChemPhvsChem 2005, 6, 2190-2196.

(23) Dresselhaus, M. S.; Dresselhaus, G.; Saito, R.; Jorio, A. Phvs. Rep 2005, 409, 47-99.

(24) De Martini, F.; Marrocco, M.; Mataloni, P.; Crescentini, L.; Loudon, R. Phvs. Rev. A 1991, 43, 2480-2497.

(25) Souza Filho, A. G.; Jorio, A.; Hafner, J. H.; Lieber, C. M.; Saito, R.; Pimenta, M. A.; Dresselhaus, G.; Dresselhaus, M. S. Phvs. Rev. B 2001, 63, 241404(R).

(26) Jorio, A.; Souza Filho, A. G.; Dresselhaus, G.; Dresselhaus, M. S.; Swan, A. K.; Ünlü, M. S.; Goldberg, B. B.; Pimenta, M. A.; Hafner, J. H.; Lieber, C. M.; Saito, R. Phvs. Rev. B 2002, 65, 155412.

(27) O'Connell, M.; Bachilo, S. M.; Huffman, C. B.; Moore, V. C.; Strano, M. S.; Haroz, E. H.; Rialon, K. L.; Boul, P. J.; Noon, W. H.; Kittrell, C.; Ma, J.; Hauge, R. H.; Weisman, R. B.; Smalley, R. E. Science 2002, 297, 593-596

(28) Hagen, A.; Steiner, M.; Raschke, M. B.; Lienau, C.; Hertel, T.; Qian, H.; Meixner, A. J.; Hartschuh, A. Phvs. Rev. Lett. 2005, 95, 197401.

(29) Misewich, J. A.; Martel, R.; Avouris, P.; Tsang, J. C.; Heinze, S.; Tersoff, J. Science 2003, 300, 783-786.

(30) Mann, D.; Kato, Y. K.; Kinkhabwala, A.; Pop, E.; Cao, J.; Wang, X.; Zhang, L.; Wang, Q.; Guo, J.; Dai, H. Nat. Nanotechnol. 2007, 2, 33-38.

NL070693I 